\title{
Soft - Partial Frequency Reuse Method for LTE-A
}

\author{
Slawomir GAJEWSKI \\ Dept. of Radio Communication Systems and Networks, Faculty of Electronics, Telecommunications and Informatics, \\ Gdansk University of Technology, Narutowicza 11/12, 80233 Gdansk, Poland \\ slagaj@eti.pg.gda.pl \\ Submitted May 18, 2016 / Accepted September 11, 2016
}

\begin{abstract}
In the paper a novel SPFR frequency reuse method is proposed which can be used for improvement of physical resources utilization efficiency in LTE-A. The proposed method combines both SFR and PFR giving the possibility of more flexible use of frequency band in different regions of a cell. First, a short study on the problem of frequency reuse in cells is discussed including bibliography overview. In next section the principle of the proposed SPFR method is described. Then the simulation model is discussed and simulation parameters are expected. In the last part, results of simulation of SPFR efficiency in comparison to known frequency reuse methods are presented. Presented results include both capacity and throughput for single connection. The proposed method eliminates main disadvantages of both SFR and PFR methods and gives significantly greater capacity of radio interface in boundary region of cells.
\end{abstract}

\section{Keywords}

Frequency reuse, LTE, SPFR, FFR, resource management, SFR, PFR, ICI reduction

\section{Introduction}

The problem of Multiple Access Interference (MAI) is one of the most important issues in mobile networks of new generation. In modern communication systems single frequency networks are typically implemented where the same frequency channel is used in each cell. But using the same channels in neighbor cells causes inter-cell interferences (ICI). Interferences decrease transmission performance, reduce overall cell capacity and destroy spectral efficiency. It is strongly important in downlink transmission from base station (BS) to mobile stations (MS), especially, when MSs are located at cell edge sometimes called boundary area of cell (BAC). In general, it's well known that in BACs high power of ICI is received from neighbor BSs. Furthermore, both the cell capacity and transmission rate are strongly reduced [1-3].

In LTE and LTE-A the Fractional Frequency Reuse (FFR) methods can be used for improving signal performance, capacity and throughput in cells. The FFR is used to intelligent signal spectrum allocation that reduces the effect of ICI on signal performance. In general, the FFR is based on the allocation of small part of available frequency band for cell-edge connections. Two main methods are known, and their modifications, called the Soft and Partial Frequency Reuse (SFR and PFR). Sometimes, the PFR is called the Strict FFR. Each of these methods has some advantages and disadvantages what determines their usefulness in practical applications. A comprehensive analysis of known frequency reuse methods is presented in survey [4] but there are many more publications about FFR.

In [5] a contribution to analytical quantification of interference cancellation and SINR (Signal to Noise and Interference Ratio) estimation in LTE is presented and a cell coverage improvement is identified when using FFR. A comparison of SFR and strict FFR was done in [6] where analytical evaluation of both methods is presented. Moreover, in [7] some modifications of PFR in which the band reserved for BAC is doubled are proposed, as a cost of some additional interference. Authors of [8] propose an interference avoidance scheme for SFR used in LTE downlink. Additionally, in [9] a mechanism that selects efficient FFR scheme based on the user throughput and user satisfaction is proposed while in [10] efficient algorithms for bandwidth and power allocation depending on user's selection are analyzed.

Interesting concepts are studied in [11] where modification of SFR to increase resource usage efficiency called Enhanced Fractional Frequency Reuse (EFFR] is proposed. It is partially based on Incremental Frequency Reuse (IFR) presented before in [12]. The IFR scheme reuses effectively frequency bands through systematic segment allocation over a cluster of adjoining cells. Some improvement of SFR is done in [13] where a number of power density levels, achieving better interference pattern and increasing data rate at cell edge is studied.

In [14] the proposal of SFR enhancement for interference management in femto-cells located inside macro-cells is discussed while in [15] resource allocation scheme that gradually varies frequency resource share with distance from BS for both macro-cells and femto-cells is analyzed.

Authors of [16] give original contribution to performance analysis of frequency planning for FFR methods due to subcarrier collisions. Moreover, in [17] some re- 
source adaptation concepts study for the case of uplink transmission using adaptive SFR is discussed. In [18] a proposal for frequency bands planning that simplifies the problem of subcarrier allocation with frequency reuse in OFDMA networks is analyzed.

Authors of [19] propose a self-organizing algorithm for sub-carrier and power allocation that achieves ICI avoidance while in [20] a new scheme for efficient location for Relay Nodes in LTE is discussed.

It's obvious that there are many modifications of FFR for improving signal performance and network capacity. In this paper basic concepts were taken into account because they are used to compare with the modified method called the Soft-Partial Frequency Reuse (SPFR).

In the case of PFR, the overall frequency band (a given radio channel) is divided into two parts. The first part is used in central area of cell (CAC) only (in each cell) and the second part is available in BAC. Additionally, the part of band reserved for BAC is divided into three parts which are allocated to different groups of cells. This minimizes the effect of ICI on signal performance and increases the transmission rate achievable in each cell of a network. But, the use of PFR increases the frequency reuse factor and decreases the capacity of a network (a cell) what is clearly presented in e.g. [2], [6]. A major advantage of PFR is approximately almost total interference isolation for celledge users. The principle of PFR is clearly shown in [2].

In the case of SFR, full frequency band can be used to all connections in CAC but only a small part of this band can be used to BAC connections. Additionally, this small part of frequency band is different for adjacent cells $[2,6]$.

In general, the SFR gives better capacity but the PFR gives better results of throughput for single connection what was presented in [2]. In PFR, the result of reservation of the part of available frequency band to its use in BAC only (excluding CAC) is large capacity reduction both in $\mathrm{CAC}$ and BAC. On the other hand, the use of SFR guarantees better values of the capacity in BAC but decreases transmission rate achieved in this area.

\section{SPFR Concept}

The main goal of this paper is to present SPFR method for FFR improvement. The main advantage of SPFR is overall cell capacity enhancement, especially, increasing the capacity of BAC. The SPFR is the method in which some features of SFR and PFR were applied. The principle of SPFR is to make decisions about the resource allocation for various MSs on the basis of users' location information in different cell regions and on the basis of the load measurement in a cell. Thus, for the realization of SPFR, MSs monitoring is necessary and dynamic creation of the location map. But, we know that MSs location information is continuously reported to BS because the same

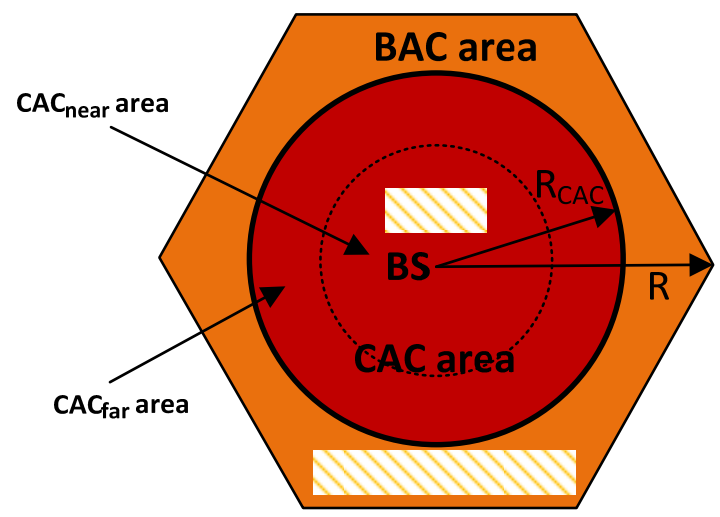

Fig. 1. A cell division into $C A C_{\text {near }}, C A C_{\text {far }}$ and $B A C$ for SPFR; $R_{\mathrm{CAC}}$ is the radius of CAC, and $R$ is the total cell radius.

problem is with users location when both SFR and PFR standardized methods are used.

For the creation of users location map a cell division into different regions is necessary as shown in Fig. 1.

In practice, the problem of SPFR parameters configuration consists in distance measurement for $\mathrm{CAC}_{\text {near }} / \mathrm{CAC}_{\text {far }} / \mathrm{BAC}$ configuration and it is basically the same problem as in the case of both SFR and PFR. Typically, configuration is made using radio path distance information. Of course, in this situation allocation of users to different regions not fully corresponds to their actual geographical location but this is the simplest way for the use of FFR methods in cells. Additionally, it is contemplated the use of location service. Then the problem of time alignment is partially resolved. The location service is available in LTE and still optimized.

In the case of SPFR users can be mapped into three different regions of a cell:

- BAC region for cell-edge users, as in PFR,

- $\mathrm{CAC}_{\text {near }}$ region for users allocated close to the BS,

- $\mathrm{CAC}_{\text {far }}$ region for users between $\mathrm{CAC}_{\text {near }}$ and BAC.

A cell radius is denoted as $R$, and it is the result of a network design. The CAC radius is denoted as $R_{\mathrm{CAC}}$ and determines the geometrical relationship between CAC and $\mathrm{BAC}$. The $R_{\text {near }}$ radius of $\mathrm{CAC}_{\text {near }}$ is designed in such a way that the $\mathrm{CAC}_{\text {near }}$ area is the same as the $\mathrm{CAC}_{\text {far }}$ area. Thus, the relationship between $R_{\text {near }}$ and $R_{\mathrm{CAC}}$ is defined as

$$
R_{\text {near }}=\frac{1}{\sqrt{2}} R_{\mathrm{CAC}} .
$$

However, it is obvious that it can be designed in another way and can be optimized due to the actual network operating conditions and geometrical aspects.

\subsection{Frequency Allocation}

In the case of SPFR, frequency band allocation is made in different way in comparison to PFR. We use three types of frequency bands depending on the place of their 


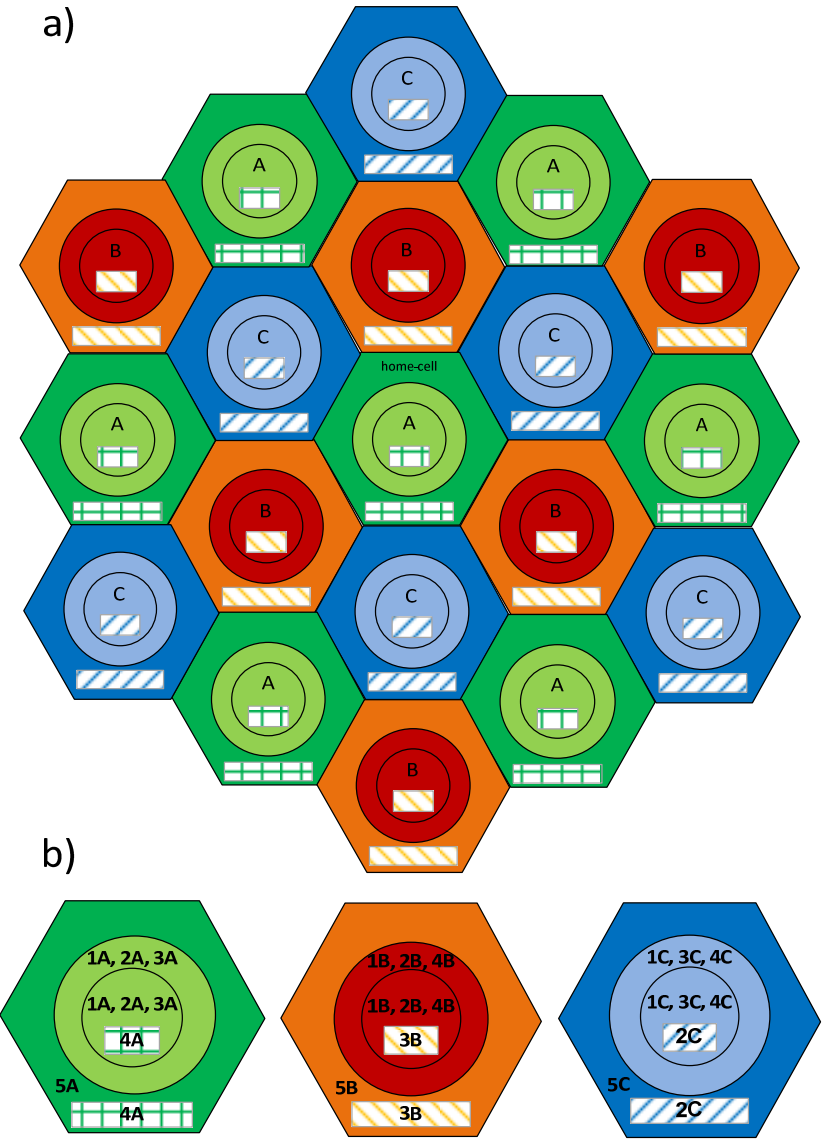

Fig. 2. General concept of frequency band allocation in SPFR: a) division into groups of cells, b) band allocation for cells from different groups.

use in CAC or BAC. Additionally, the way of band allocation depends on groups of cells (or sectors) as shown in Fig. 2. These groups are denoted as A, B and C.

Both in PFR and SPFR, the $10 \%$ of the total channel band is allocated to BAC region only. It can be used in a single group of cells and this band is different for each group. Note that signals in these bands are transmitted at increased power (e.g. $3 \mathrm{~dB}$ over the power of signals transmitted at the rest of a band, excluding pilot signals). The $20 \%$ of the total band is an unused band and it can be used in other groups of cells only.

The novelty is that for BAC we can use not only the small part of total band mentioned before (as in PFR) but, additionally, it is possible to use some soft-band for both $\mathrm{BAC}$ and CAC. This soft-band equals to $20 \%$ of the total band and it is different for various groups of cells. This is similar to the SFR but the difference is that the soft-band can be used for users allocated close to the serving BS in the $\mathrm{CAC}_{\text {near }}$ region only (not in $\mathrm{CAC}_{\text {far }}$ ), and for users in the BAC.

It is presented in Fig. 3 where the soft-band is denoted as $4 \mathrm{~A}, 3 \mathrm{~B}$ or $2 \mathrm{C}$ depending on the group of cells.

The remaining part of band which is $50 \%$ of the total band is allocated for CAC area only and it can be used both in $\mathrm{CAC}_{\text {near }}$ and $\mathrm{CAC}_{\text {far }}$ regions. The first $10 \%$ part of this
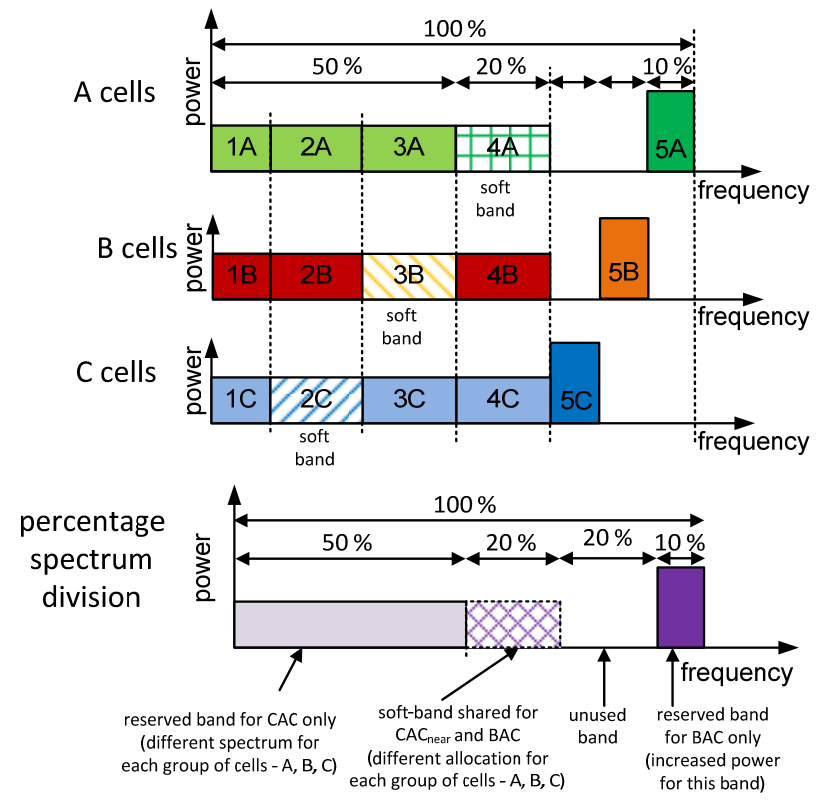

Fig. 3. Detailed SPFR spectrum allocation for different groups of cells.

band is the same for each group of cells and this is denoted in Fig. 3 as 1A, 1B, and 1C. Additionally, we can use 2 parts of $20 \%$ of the total band in the CAC region. For the A group of cells, these parts are $2 \mathrm{~A}$ and $3 \mathrm{~A}$ bands, and for the $\mathrm{B}$ group these are $2 \mathrm{~B}$ and $4 \mathrm{~B}$, and for the $\mathrm{C}$ group these are $3 \mathrm{C}$ and $4 \mathrm{C}$ bands. So, in each $\mathrm{CAC}$ region we can use the part of band reserved for the use in a given group of cells and this band is $50 \%$ of the total band. And, additionally, we can use the soft-band which is $20 \%$ of the total band but it can be used in $\mathrm{CAC}_{\text {near }}$ part of CAC only and this band is shared between $\mathrm{CAC}_{\text {near }}$ and BAC. Furthermore, for BAC we can use both the reserved-band (10\% of the total band) and the soft-band.

The use of soft-band in the CAC causes additional ICI in a network compared to PFR. But, due to its use in $\mathrm{CAC}_{\text {near }}$ zone only, additional ICI are not critical. While it gives large gain in capacity achievable in BAC region compared to strict PFR. The decision to the use of these parts is taken dynamically by a system and it is dependent on a cell load. The load is measured for both the bands allocated to $\mathrm{CAC}$ and $\mathrm{BAC}$ regions and results from the actual frequency band occupation.

\subsection{The Principle of SPFR}

At the beginning, the soft-band is dedicated to users allocated in the $\mathrm{CAC}_{\text {near }}$ region only. If the load in the frequency band allocated to BAC-users grows then the softband is progressively allocated to these connections, if necessary.

The power of signals transmitted in soft-band at BAC is not-increased compared to the power of signals transmitted in bands reserved for BAC only. Thus, the best situation is when soft-band is allocated to MSs located close the $\mathrm{CAC}_{\text {far }}$ region (the boundary between $\mathrm{CAC}_{\text {far }}$ and 
BAC). While users located at cell edge should better use the bands reserved for BAC which are denoted as 5A, 5B or $5 \mathrm{C}$. Note that it is not critical and depends on load and signal quality measurements. In simulations it was seen that the SINR measured for signals in soft-band at BAC and reserved-band at BAC do not differ significantly in most situations, and sometimes the SINR is better for softband, sometimes for reserved-band.

On the other hand, the soft-band can be used in $\mathrm{CAC}_{\text {near }}$ and $\mathrm{BAC}$ only (not in $\mathrm{CAC}_{\text {far }}$ ). It takes place because the use of soft-band in $\mathrm{CAC}_{\text {far }}$ causes additional ICI interferences to other groups of cells. So, the isolation between $\mathrm{BAC}$ and $\mathrm{CAC}_{\text {near }}$ regions is recommended in each cell. For instance, the use of the $4 \mathrm{~A}$ soft-band in the $\mathrm{A}$ group of cells causes ICI both to $4 \mathrm{~B}$ and $4 \mathrm{C}$ what we can see in Fig. 2 and Fig. 3. If the soft-band was used in $\mathrm{CAC}_{\text {far }}$ then the distance from $\mathrm{BS}$ to $\mathrm{MSs}$ located in $\mathrm{CAC}_{\text {far }}$ regions is less compared to the situation when $4 \mathrm{~A}, 4 \mathrm{~B}$, and $4 \mathrm{C}$ bands are used in $\mathrm{CAC}_{\text {near }}$ regions only. The result of this is additional ICI received in $\mathrm{CAC}_{\text {far }}$ regions in each group of cells, received signals quality deterioration and throughput reduction. Thus, the use of soft-band for $\mathrm{CAC}_{\text {near }}$ and for $\mathrm{BAC}$ is recommended but the power of signals in soft-band should not be increased when allocated to BAC.

The use of soft-band in BAC regions depends on the load measured in 5A, 5B and 5C bands. The expanded soft-band is used if the load increases over $70 \%$. Additionally, the soft-band is allocated in different way for each group of cells as we can see in Fig. 2 and Fig. 3.

In conclusion, we can use in a cell the bands denoted in Fig. 3 as:

- $1 \mathrm{~A}, 1 \mathrm{~B}$ and $1 \mathrm{C}$ which are the same for each group of cells (A, B and $\mathrm{C}$ ), and these bands are reserved for both $\mathrm{CAC}_{\text {near }}$ and $\mathrm{CAC}_{\text {far }}$,

- $4 \mathrm{~A}, 3 \mathrm{~B}$ or $2 \mathrm{C}$ (depending on the group of cells) which can be used as soft-bands both in $\mathrm{CAC}_{\text {near }}$ and BAC,

- 2A, 3A, 2B, 4B, 3C, 4C which can be used in CAC only,

- 5A, 5B, and 5C which are reserved for BAC only.

One can see that a different way of soft-band placing in each group of cells gives some reduction of ICI received in various cells. It's clear that ICI are not completely eliminated. But we see that in SPFR the band allocated to MSs placed in BAC can be soft-expanded. The expansion can be made using the part of band allocated for both CAC and BAC called the soft-band. And it is a major difference between SPFR and strict PFR.

\section{Simulation Results}

The simulation model allows research of throughputcoverage characteristics of the OFDMA-based LTE network (see [2]). Simulation process is based on Monte Carlo method. The aim of simulation is to prove that the use of SPFR method allows increasing the efficiency of known frequency reuse methods.

\subsection{Simulation Model}

The network model was implemented as a set of cells including the central cell (denoted as the home-cell in Fig. 2) and two tiers of surrounding cells which introduce ICI. Each cell is divided into $\mathrm{CAC}_{\text {near }}, \mathrm{CAC}_{\text {far }}$ and $\mathrm{BAC}$ areas. The $R_{\mathrm{CAC}}$ radius sets the geometrical relationship between CAC and BAC in each cell. Thus, $R_{\mathrm{CAC}}$ can be changed during simulation from $0.1 R$ to $0.9 R$. Each MS is located using geometrical relations between BSs and MSs and it defines its placing to different cells and regions of the home-cell.

The network simulation is based on random process of mobile stations (MS) placing in cells, estimation of ICI received by MSs at different locations and on the estimation of signal quality given by SINR. On the basis of actual SINR, we can estimate throughput-coverage characteristics for connections established in a cell. If a time of simulation is sufficiently long, then we can estimate average value of throughput for connections and evaluate real network capacity. The home-cell connections are observed because this cell allows us to estimate the total power of ICI. So, the home-cell is a reference cell in overall simulation process. The distribution of users in cells is uniform. ErcegGreenstein propagation loss model [21] for suburban environment was implemented. Slow fadings were simulated using the log-normal distribution.

Received signals are analyzed in the spatial grid where we have a large number of points represented by small squares having sides equal to $2 \mathrm{~m}$. In Fig. 4 we can see example results of SINR visualization for SFR.

The basic set of simulation parameters is presented in Tab. 1.

As we can see in the next sections, for better interpretation of results, we took into account the distance $d / R$ from $\mathrm{BS}$ to $\mathrm{MS}$, normalized by $R$ (maximum cell radius)
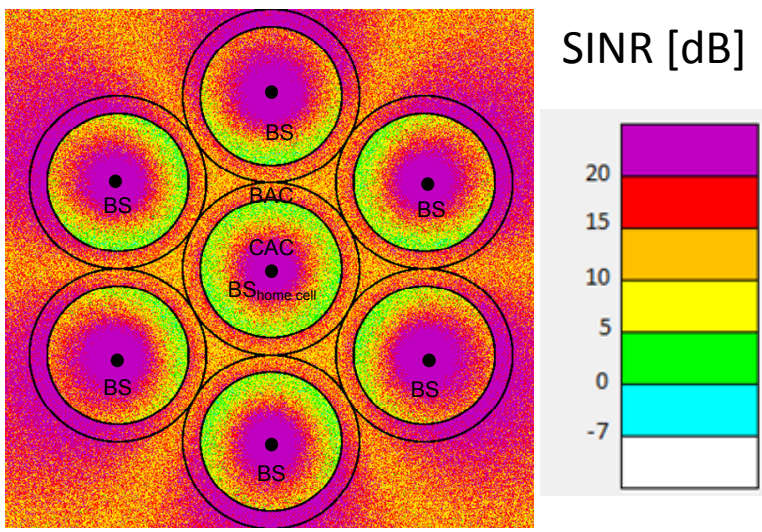

Fig. 4. Example of SINR distribution in modeled network; the home-cell and single tier of adjacent cells are shown. 


\begin{tabular}{|c|c|}
\hline Parameter & Setting \\
\hline Reuse methods & PFR, SFR, SPFR \\
\hline Frequency band & $10 \mathrm{MHz}$ \\
\hline Central frequency & $2.6 \mathrm{GHz}$ \\
\hline Maximum BS power & $30 \mathrm{dBm}$ \\
\hline Transmission type & $\begin{array}{c}\text { Single antenna } \\
\text { connection }\end{array}$ \\
\hline Designed cell radius $R$ & $400 \mathrm{~m}$ \\
\hline Propagation model & Erceg-Greenstein \\
\hline Antenna type & Omni-directional \\
\hline Slow fluctuations & $\begin{array}{c}\text { Log-normal distribution } \\
(8 \mathrm{~dB} \text { signal deviation })\end{array}$ \\
\hline Scheduling & Proportional Fair \\
\hline Users distribution & Uniform \\
\hline
\end{tabular}

Tab. 1. Set of basic simulation parameters.

and the throughput for single connection $C_{\mathrm{MS}} / C_{\mathrm{MSmax}}$ normalized by maximum throughput achievable in a network for this connection. As well as the overall cell capacity, $C / C_{\max }$ is normalized by the maximum capacity available in a cell. So, both the $C_{\mathrm{MS}} / C_{\mathrm{MSmax}}$ and $C / C_{\max }$ are observed at various distances $d / R$.

\subsection{Simulation Results of Throughput for Single Connection}

This simulation scenario corresponds to the case of low-loaded network (in home-cell) when there is a possibility of the transmission of signals for single connection with the transmission rate as high as possible in a radio interface. It means that other home-cell connections do not affect transmission rate for analyzed, single connection.

The throughput for single connection is observed for downlink transmission from BS to the reference MS. Three methods were evaluated: SFR, PFR in classic form and the proposed SPFR. MS is observed in different locations and at various distances from BS in home-cell. So, we receive signals with different SINR and various power of ICI from adjacent cells.

The maximum throughput $C_{\mathrm{MSmax}}$, used to normalizations, is calculated for single connection at different distances from home BS. This it is the maximum throughput achievable for best CQI (Channel Quality Indicator) in frequency band available at a given distance from BS (i.e. available for a given MS). Thus, $C_{\mathrm{MSmax}}$ is different for various distances (MS locations) and, in general, it is not maximum throughput available in a cell and it cannot be associated with a cell capacity. Thus, presented charts provide the information how the throughput is reduced at different distances from BS compared to the maximum throughput available at this distance for the best CQI.

The maximum transmitted power of BS is sufficient for the best transmission performance estimated by SINR for low ICI. So, during simulations the power does not limit the throughput, excluding situations of very large ICI received (i.e. in BAC). In modeled case, the network is not capacity limited what means that theoretical capacity is available, and radio interface is not fully-loaded by users.
These simulation conditions include the type of connection, possible coding-modulation scheme (dependent on CQI), maximum BS power, signal loss and fadings, etc.

The situation is possible when ICI growth causes the situation in which BS power cannot be sufficient for interference compensation. Thus, SINR is reduced and throughput is decreased due to different CQI as in a real LTE-A network [2]. Moreover, the analyzed throughput for single connection can be reduced if capacity is too small in the observed area (i.e. ICI cannot be compensated by the transmitted BS power). It's obvious that the throughput is greater when the MS is located close to the BS in the home-cell and decreases when it moves away due to ICI growth. However, when MS moves to BAC depending on the simulated $R_{\mathrm{CAC}}$, the throughput strongly grows because the power of ICI is relatively small in this region.

It was assumed that the MS is located in CAC when its geometrical distance to the $\mathrm{BS}$ is between $0<d / R \leq R_{\mathrm{CAC}} / R\left(0<d \leq R_{\mathrm{CAC}}\right)$. At greater distance, from $R_{\mathrm{CAC}} / R<d / R \leq 1 \quad\left(R_{\mathrm{CAC}}<d \leq R\right)$, the $\mathrm{MS}$ is located in BAC. Additionally, in the CAC area MSs are mapped to $\mathrm{CAC}_{\text {near }}$ or $\mathrm{CAC}_{\text {far }}$ areas, depending on their actual location, taking into account the $R_{\text {near }}$ radius.

Simulation results are presented in Fig. 5-9, as the throughput $C_{\mathrm{MS}} / C_{\mathrm{MSmax}}$ available for single connection normalized by its maximum value, appointed for different $R_{\text {CAC }} / R$ configurations.

As we can see, the achieved throughput for single connection is the greatest for PFR. It is possible because the simulated network is not fully-loaded in the presented experiment. As previously mentioned, it takes place because throughput for single connection is the strength point of the PFR method, especially, compared to SFR (see [2]).

On the other hand, we know that for the SFR the throughput in BAC is small. This is the main SFR disadvantage. One can see that the use of PFR gives better stability of throughput for single connection compared to other methods - especially SFR. The reservation of ICIresistant frequency band guarantees higher values of SINR especially in the BAC region.

As we see, SPFR gives a little worse results of throughput for single connection achieved in BAC, compared to PFR. However, deterioration in BAC is from a dozen $\%$ to approximately $20 \%$. This is the price we must pay for the modification of the strict PFR. At the same time, it should be noted that the throughput is much greater in comparison to SFR. So, we can notice that the SPFR strongly reduces the main disadvantage of SFR in the BAC region while deterioration of throughput for PFR is not too large.

In the $\mathrm{CAC}$ region, moreover, we have approximately the same results of throughput for both PFR and SPFR. So, mentioned earlier deterioration concerns the BAC region only. Note, that in this case both PFR and SPFR methods give better results than SFR. 


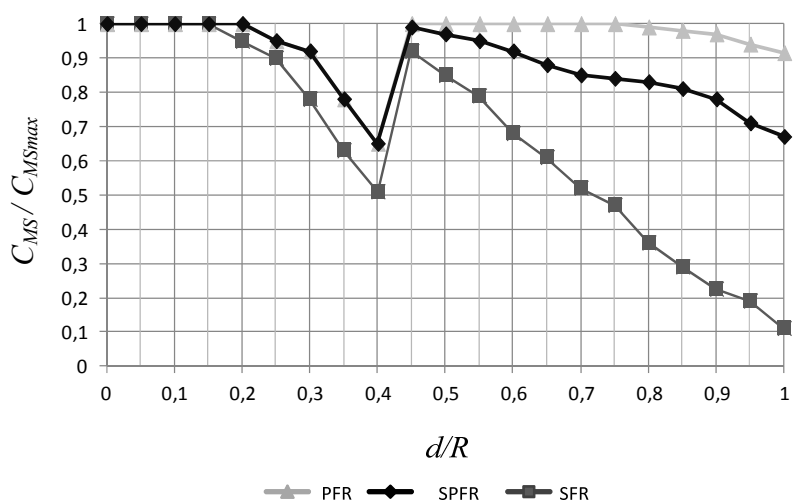

Fig. 5. Maximum throughput for single connection for PFR, SPFR and SFR; $R_{\mathrm{CAC}}=0.4 R$.

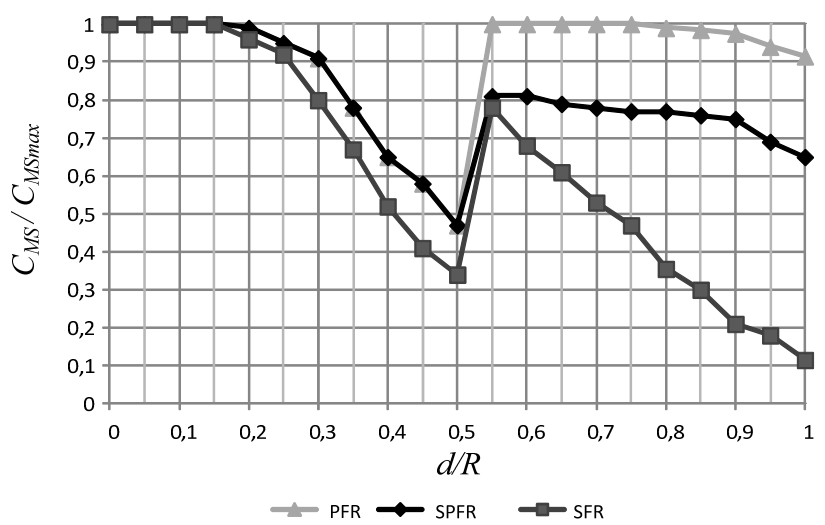

Fig. 6. Maximum throughput for single connection for PFR, $\mathrm{SPFR}$, and SFR; $R_{\mathrm{CAC}}=0.5 R$.

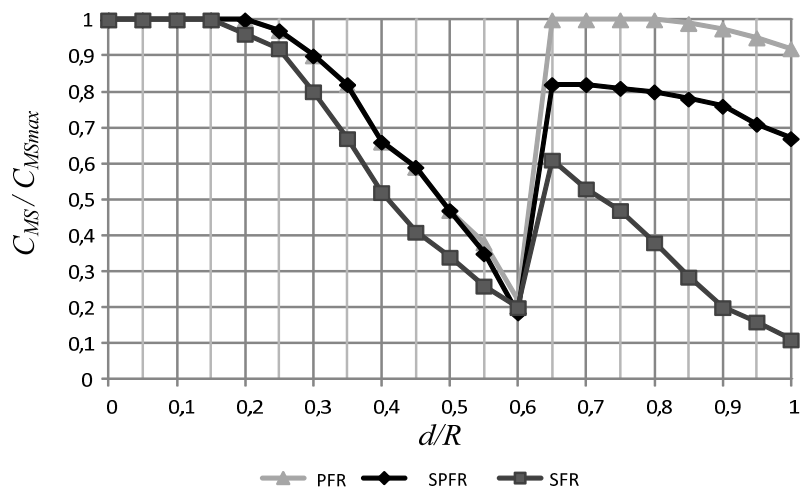

Fig. 7. Maximum throughput for single connection for PFR, SPFR, and SFR; $R_{\mathrm{CAC}}=0.6 R$.

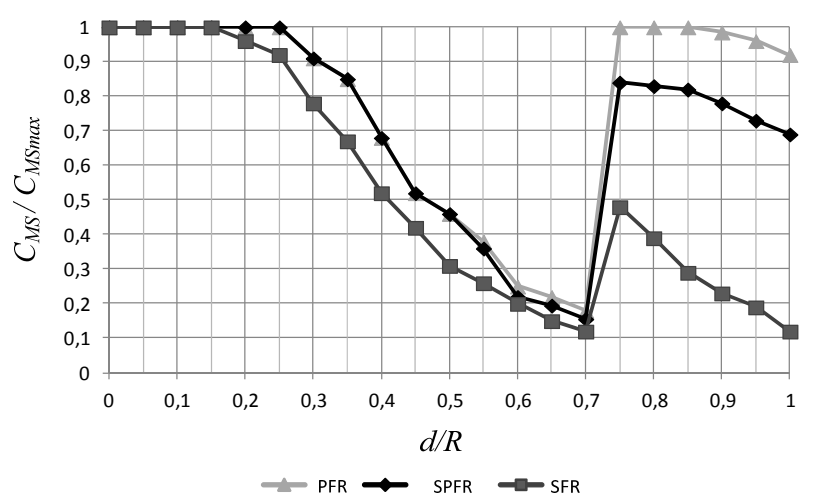

Fig. 8. Maximum throughput for single connection for PFR, SPFR, and SFR; $R_{\mathrm{CAC}}=0.7 R$.

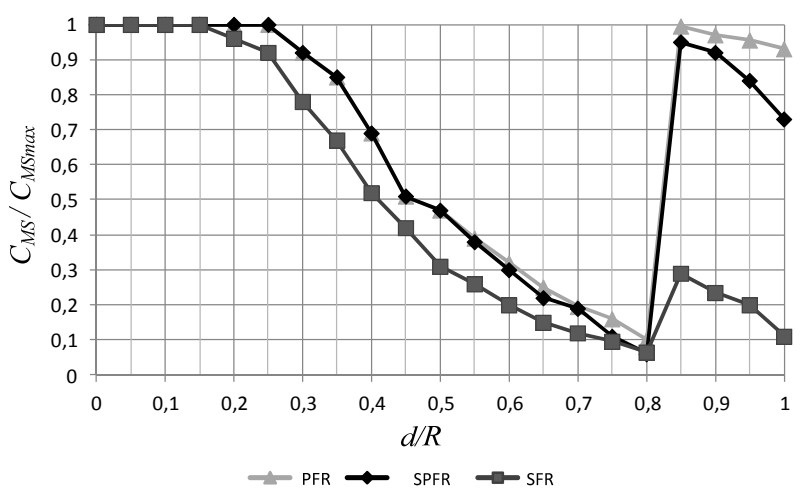

Fig. 9. Maximum throughput for single connection for PFR, $\mathrm{SPFR}$, and SFR; $R_{\mathrm{CAC}}=0.8 R$.

As we can see in presented figures, the $R_{\mathrm{CAC}}$ radius plays a great role in the design process and achievable transmission efficiency. The use of large $R_{\mathrm{CAC}}$ is not recommended because throughput for single connection is rather better for $R_{\mathrm{CAC}}=0.5 R$ or $R_{\mathrm{CAC}}=0.6 R$ than for larger values. On the other hand, we can see that BAC should not be too small in comparison to CAC. It is strongly important not only for uniform users' distribution in a cell. We know that in most cases the number of users in BAC is greater than their number in CAC. So, rather $R_{\mathrm{CAC}}$ radiuses less than $0.7 R$ are preferred. Additionally, we should not use too small $R_{\mathrm{CAC}}$ due to high capacity reduction as we can see in the next section.

\subsection{Cell Capacity Analysis}

The Shannon capacity bound in AWGN channel, applied to practical LTE system implementation was clearly considered in [1], [3]. So, the spectral efficiency can be estimated using the formula

$$
S_{\text {eff, } \max }=B_{\text {eff }} \sum_{k=1}^{\min \left(l_{\text {Ix }}, l_{\text {Rx }}\right)} \log _{2}\left(1+\frac{\operatorname{SINR} R_{k}}{\operatorname{SINR} R_{\text {eff }, k}}\right)[\text { bits } / \mathrm{s} / \mathrm{Hz}]
$$

where:

- $l_{\mathrm{Tx}}$ and $l_{\mathrm{Rx}}$ are the numbers of transmit and receive antennas, respectively (when MIMO is used),

- $S I N R_{k}$ is signal-to-interference and noise ratio resulting from the $k$-th spatial subchannel,

- $S I N R_{\text {eff }, k}$ is the factor of SINR implementation efficiency for the $k$-th subchannel [3],

- $B_{\text {eff }}$ is bandwidth efficiency factor of LTE [3].

Typical value of $B_{\text {eff }}$ is 0.9 as was explained in [3]. It is obvious that full SINR efficiency is not possible in LTE due to the limited code block length and it may be determined by the simulation research. Additionally, the bandwidth efficiency is reduced by the overhead of the use of cyclic prefix and the pilot assisted channel estimation as well as other overheads.

In practice, the capacity of LTE air interface can be understood as the maximum throughput available for all users in a given frequency channel, at a given moment of 
time. In OFDMA based radio interface the channel is composed of some number of subcarriers and resource blocks. So, the capacity depends on channel bandwidth, the number of subcarriers, resource elements and blocks as well as on the number of OFDM symbols allocated in a time slot (frame). Also, the capacity depends on coding rate and modulation type, i.e. on the number of bits per modulation symbol. Furthermore, it depends on the number of transmit and receive antennas if the MIMO (Multiple Input Multiple Output) technique is used.

A major capacity estimation problem results from the determination of the number of available physical resource blocks for a given user from the plan of the structure of data transmitted in a single time slot as well as from the number of OFDM symbols available for this user with the exception of control data overhead. When the number of available resource blocks has been planned, and data structure has been designed, we may to estimate the total capacity $C_{\mathrm{p}}$ [bps] as maximum throughput available for all users. Thus, the capacity can be found using the formula

$$
C_{\mathrm{p}}=n_{\mathrm{b}} \eta_{\text {cod }} k_{\mathrm{MIMO}} l_{\mathrm{SC} \mathrm{RB}} v_{\text {loss }} l_{\text {symb }} / T_{\text {sub }}
$$

where:

- $n_{\mathrm{b}}[\mathrm{b} / \mathrm{symbol}]$ is the number of bits dependent on modulation type (QPSK: 2 bits per symbol, 16QAM: 4 bits per symbol, 64QAM: 6 bits per symbol),

- $\eta_{\text {cod }}$ is a channel coding rate,

- $k_{\mathrm{MIMO}}$ is the factor of growth of spectral efficiency when MIMO is used (corresponds to the number of transmit and receive antennas), $k_{\mathrm{MIMO}}=1$,

- $l_{\mathrm{SC} \mathrm{RB}}$ is the number of subcarriers assigned for a given service in an available frequency band,

- $l_{\text {symb }}$ is the number of OFDM symbols assigned for a given service in a single subframe,

- $T_{\text {sub }}=1 \mathrm{~ms}$ is the subframe time duration,

- $v_{\text {loss }}-$ the loss factor resulting from the use of certain OFDM symbols by the control data, i.e. reference symbols et al. [3] (minimum 2 symbols per subframe; thus $v_{\text {loss }}=0.86$ ).

In presented simulations, the capacity is calculated as the maximum throughput available for all connections in home-cell at a given distance from BS to MS in overall frequency band. Thus, the simulation supposes that the capacity at a given distance is calculated for all terminals located at this distance. The capacity is different for users located in CAC and BAC areas at various places because it depends on the received SINR and available frequency band. Moreover, the SINR determines achievable CQI which in turn sets available coding-modulation scheme and throughput. Additionally, it is different for various frequency reuse methods.

On the other hand, the overall cell capacity can be calculated as the sum of throughputs of all users which depends on many random variables. This method of capac- ity estimation gives the information about overall cell performance and it does not give the clear information how ICI affects the capacity in different regions of a cell. Thus, this information is much less valuable for performance evaluation of frequency reuse methods compared to the analysis of capacity at different distances from BS.

As mentioned before, the estimated capacity $C$ is normalized by the maximum capacity $C_{\max }$ available in a cell achieved for the best CQI at a given distance $d / R$ in overall frequency band. The $C$ and $C_{\max }$ capacities as well as $C_{\mathrm{MS}}$ and $C_{\mathrm{MSmax}}$ throughputs for single connection are calculated using (3). Thus, the method of calculation is similar but the difference is that both the maximum capacity and maximum throughput for single connection are calculated for different conditions.

The capacity analysis includes the effect of capacity loss due to SINR variability. As we can see, the capacity depends on the degree of reduction of available frequency band due to the use of different frequency reuse methods (SFR, PFR or SPFR), and depends on cell region type $\left(\mathrm{CAC}_{\text {near }}, \mathrm{CAC}_{\text {far }}\right.$ and $\left.\mathrm{BAC}\right)$. Thus, presented charts provide the information how capacity $C$ is reduced at different distances from BS compared to maximum capacity $C_{\max }$ available for the best CQI in overall frequency band.

The normalized capacity is tested for different locations of many terminals in analyzed regions of cells but it is estimated for different distances. For instance, if $C / C_{\max }=0.6$ for $d / R=0.35$ then the information is that if a terminal is at this distance then the maximum achievable capacity for its connection is 0.6 and no more, even if there are no more connections in the home-cell. It means that the capacity is not limited by other connections but it is bandlimited and ICI-limited only. The sense of this is that the way of capacity estimation can be reasonably unified for all FFR methods, and comparison of their performance in such a way is reliable.

As we can see in Fig. 10-14, better capacity can be achieved for $R_{\mathrm{CAC}}=0.5 R$ or $0.6 R$ than for greater $R_{\mathrm{CAC}}$. It is similar to simulation results achieved for the throughput for single connection. We can see that the capacity gain for BAC is very large when SPFR method is implemented. The use of SPFR gives, in general, more than 2 times greater capacity in BAC in comparison to both PFR and SFR.

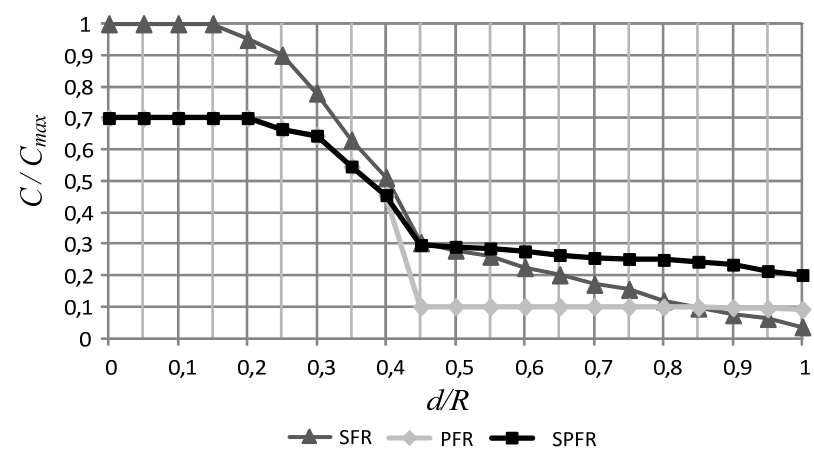

Fig. 10. Normalized capacity $C / C_{\max }$ for PFR, SPFR, and SFR; $R_{\mathrm{CAC}}=0.4 R$. 


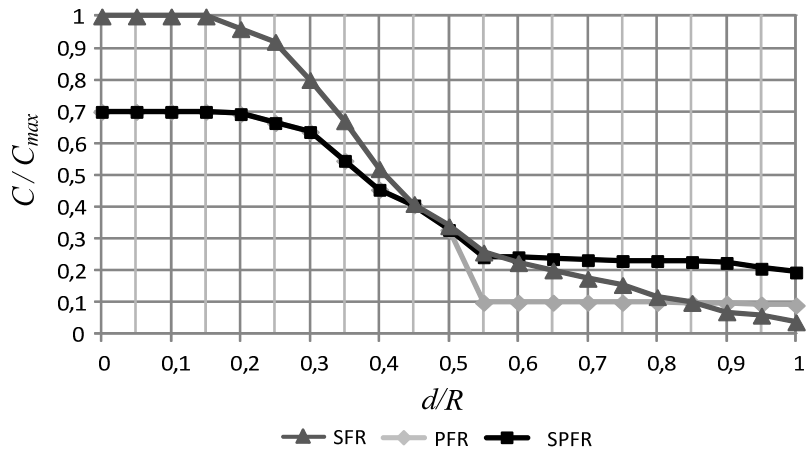

Fig. 11. Normalized capacity $C / C_{\max }$ for PFR, SPFR, and SFR; $R_{\mathrm{CAC}}=0.5 R$.

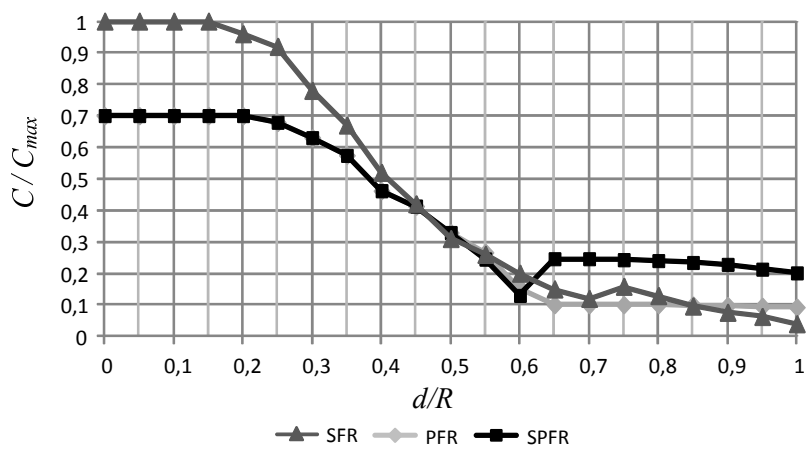

Fig. 12. Normalized capacity $C / C_{\max }$ for PFR, SPFR, and SFR; for $R_{\mathrm{CAC}}=0.6 R$.

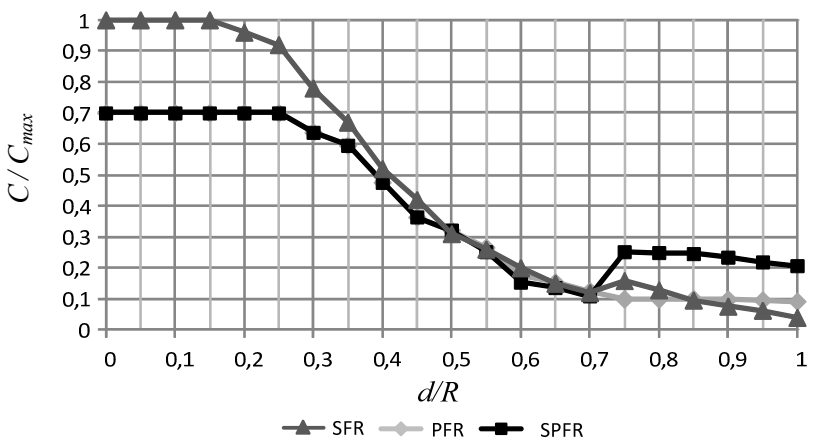

Fig. 13. Normalized capacity $C / C_{\max }$ for PFR, SPFR, and SFR; $R_{\mathrm{CAC}}=0.7 R$.

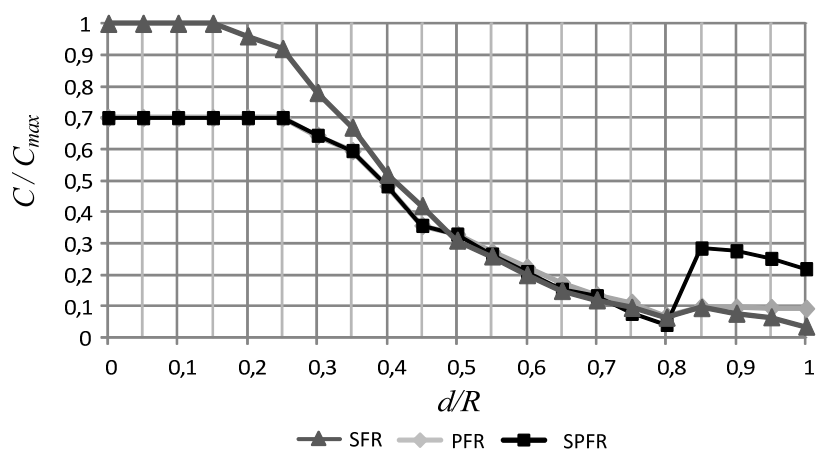

Fig. 14. Normalized capacity $C / C_{\max }$ for the PFR, SPFR and $\mathrm{SFR} ; R_{\mathrm{CAC}}=0.8 R$.

Both PFR and SPFR give capacity reduction in CAC compared to SFR due to band limitation. But it is obvious that capacity growth in $\mathrm{BAC}$ is much more important than its reduction in CAC. For SPFR the largest capacity in BAC is observed when $R_{\mathrm{CAC}}=0.5 R$. Additionally, the use of SPFR does not degrade the capacity in CAC more than PFR.

Of course, the presented results strongly depend on the designed $R_{\mathrm{CAC}}$ and it should be taken into account during the network design process and its optimization.

Note, that it is possible to reach the maximum value of $C / C_{\max }=1$ because the capacity depends on the reported CQI which, additionally, depends on SINR. If the CQI is of the maximum value then the system uses the best performance coding-modulation scheme. Thus, the capacity and throughput are maximal. In simulations, in this case SINR fluctuations were observed but SINR was greater than its value guaranteeing the transmission with the best coding-modulation scheme what we can see in Fig. 4.

In general, the overall cell capacity for PFR is less than the capacity for SPFR and it may be even more than two times greater for the BAC region. We see that SPFR significantly eliminates a major disadvantage of PFR. However, the price for this is some deterioration of throughput for single connection.

\section{Conclusions}

From the presented considerations we can see that the SPFR method significantly improves the BAC capacity compared to both strict PFR and SFR. It is very important because low capacity in BAC is the main disadvantage of these methods. The cost of this is some reduction of the available throughput for single connection compared to strict PFR and some reduction of the CAC capacity compared to SFR. But, the CAC capacity is approximately the same as achieved for PFR, and the throughput is much greater than the throughput in BAC achieved for SFR.

So, we can see that SPFR allows eliminating the capacity degradation in BAC regions of cells. In general, the results show more than twofold increase of capacity in BAC at the cost of a relatively small reduction of throughput for a single connection compared to PFR.

In conclusion, therefore, we can say that the main features of SPFR are:

- Not less than twofold increasing the total BAC capacity compared to both PFR and SFR,

- Decreasing of throughput for single connections in BAC compared to PFR which does not exceed $20 \%$,

- Greater throughput for single connections in BAC in comparison to the use of SFR,

- Little growth of algorithm implementation and frequency planning complexity,

- The need for monitoring of MS location in different cell regions although this is performed in BSs, and some complications in algorithm configuration due to problems with BS-MS distance measurement. 
Thus, a major conclusion is that SPFR is a good candidate for frequency reuse method implemented in OFDMA-based networks of $4 \mathrm{G}$ and $5 \mathrm{G}$. It can be used to improve the radio access network performance as a good compromise between SFR and PFR.

\section{References}

[1] SCHOENEN, R., ZIRWAS, W., WALKE, B. W. Capacity and coverage analysis of 3GPP-LTE multihop deployment scenario. In Proceedings of IEEE International Conference on Communications, ICC Workshops. Beijing (China), 2008, p. 31-36. DOI: 10.1109/ICCW.2008.11

[2] GAJEWSKI, S. Throughput-coverage characteristics for soft and partial frequency reuse in the LTE downlink. In Proceedings of 36th International Conference on Telecommunications and Signal Processing (TSP 2013). Rome (Italy), 2013, p. 199-203. DOI: 10.1109/TSP.2013.6613919

[3] MOGENSEN, P., WEI, N., KOVACS, I., et al. LTE capacity compared to the Shannon bound. In Proceedings of IEEE 65th Vehicular Technology Conference (VTC 2007-Spring). Dublin (Ireland), 2007, p. 1234-1238. DOI: 10.1109/VETECS.2007.260

[4] PORJAZOSKI, M., POPOVSKI, B. Analysis of intercell interference coordination by fractional frequency reuse in LTE. In Proceedings of International Conference on Software, Telecommunications and Computer Networks (SoftCOM). Dubrovnik (Croatia), 2010, p. 160-164.

[5] MAO, X., MAAREF, A., TEO, K. H. Adaptive soft frequency reuse for intercell interference coordination in SC-FDMA based 3GPP LTE uplinks. In Proceedings of IEEE Global Telecommunications Conference (GLOBECOM). New Orleans (USA), 2008, p. 1-6. DOI: 10.1109/GLOCOM.2008.ECP.916

[6] GHAFFAR, R., KNOPP, R. Fractional frequency reuse and interference suppression for OFDMA networks. In Proceedings of 8th International Symposium on Modeling and Optimization in Mobile, Ad Hoc and Wireless Networks (WiOpt). Avignon (France), 2010, p. 273-277.

[7] RAHMAN, M., YANIKOMEROGLU, H., WONG, W. Interference avoidance with dynamic inter-cell coordination for downlink LTE system. In Proceedings of IEEE Wireless Communications and Networking Conference (WCNC). Budapest (Hungary), 2009, p. 1-6. DOI: 10.1109/WCNC.2009.4917761

[8] BILIOS, D., BOURAS, C., KOKKINOS, V., et al. Optimization of fractional frequency reuse in long term evolution networks. In Proceedings of IEEE Wireless Communications and Networking Conference (WCNC). Paris (France), 2012, p. 1853-1857. DOI: 10.1109/WCNC.2012.6214087

[9] XIE, Z., WALKE, B. Frequency reuse techniques for attaining both coverage and high spectral efficiency in OFDMA cellular systems. In Proceedings IEEE Wireless Communications and Networking Conference (WCNC). Sydney (Australia), 2010, p. 1-6. DOI: $10.1109 /$ WCNC.2010.5506110

[10] NOVLAN, T. D., GANTI, R. K., GHOSH, A., ANDREWS, J. G. Analytical evaluation of fractional frequency reuse for OFDMA cellular networks. IEEE Transactions on Wireless Communications, 2011, vol. 10, no. 12, p. 4294-4395. DOI: 10.1109/TWC.2011.100611.110181

[11] HINDIA, M. N., KHANAM, S., REZA, A., W., et al. Frequency reuse for 4G technologies: A survey. In Proceedings of the 2nd International Conference on Mathematical Sciences \& Computer Engineering (ICMSCE 2015). Langkawi (Malaysia), 2015.
[12] KRASNIQI, B., MACLENBRAUKER, C. F. Efficiency of partial frequency reuse in power used depending on user's selection for cellular networks. In Proceedings of IEEE 22nd International Symposium on Personal Indoor and Mobile Radio Communications (PIMRC). Toronto (Canada), 2011, p. 268-272. DOI: 10.1109/PIMRC.2011.6139963

[13] YANG, X. A multilevel soft frequency reuse technique for wireless communication systems. IEEE Communications Letters, 2014, vol. 18 , no. 11, p. 1983-1986. DOI: 10.1109/LCOMM.2014. 2361533

[14] SELIM, M. M., EL-KHAMY, M., EL-SHARKAWY, M. Enhanced frequency reuse schemes for interference management in LTE femtocell networks. In Proceedings of International Symposium on Wireless Communications Systems (ISWCS). Paris (France) 2012, p. 326-330. DOI: 10.1109/ISWCS.2012.6328383

[15] ElayOuBI, S. E., BEN HADDADA, O., FOURESTIE, B. Performance evaluation of frequency planning schemes in OFDMA-based networks. IEEE Transactions on Wireless Communications, 2008, vol. 7, no. 5, p. 1623-1633. DOI: 10.1109/TWC.2008.060458

[16] ALI, S. H., LEUNG, V. C. M. Dynamic frequency allocation in fractional frequency reused OFDMA networks. IEEE Transactions on Wireless Communications, 2009, vol. 8, no. 8, p. 4286-4295. DOI: 10.1109/TWC.2009.081146

[17] KIM, K. T., OH, S. K. An incremental frequency reuse scheme for an OFDMA cellular system and its performance. In Proceedings of IEEE Vehicular Technology Conference (VTC 2008-Spring). Marina Bay (Singapore), 2008, p. 1504-1508. DOI: 10.1109/VETECS.2008.352

[18] STOLYAR, A. L., VISWANATHAN, H. Self-organizing dynamic fractional frequency reuse in OFDMA systems. In Proceedings of the IEEE 27th Conference on Computer Communications (INFOCOM). Phoenix (USA), 2008. DOI: 10.1109/INFOCOM.2008.119

[19] ALDHAIBANI, J. A., YAHYA1, A., AHMAD, A. B. Optimizing power and mitigating interference in LTE-A cellular networks through optimum relay location. Elektronika ir Elektrotechnika, 2014, vol. 20, no. 7, p. 73-79. DOI: 10.5755/j01.eee.20.7.3379

[20] KAWSER, M. T., ISLAM, M. R., AHMED, K. I., KARIM, M. R., SAIF, J. B. Efficient resource allocation and sectorization for fractional frequency reuse (FFR) in LTE femtocell systems. Radioengineering, 2015, vol. 24, no. 4, p. 940-947. DOI: $10.13164 /$ re. 2015.0940

[21] ERCEG, V., GREENSTEIN, L. J., TJANDRA, S. Y., et al An empirically based path loss model for wireless channels in suburban environments. IEEE Journal on Selected Areas in Communications, 1999, vol. 17, no. 7, p. 1205-1211. DOI: $10.1109 / 49.778178$

\section{About the Author ...}

Slawomir GAJEWSKI received his Ph.D. degree in Radio Communication Systems from Gdansk University of Technology (Poland) in 2004. Now, he works in the Dept. of Radio Communication Systems and Networks, Faculty of Electronics, Telecommunications and Informatics in Gdansk. He is an author of more than 140 publications in radio communications published in Poland and in many countries. He is a member of scientific committees and reviewer of many international conferences and journals (IEEE, IARIA, and Mosharaka). He is the TCP member of IEEE Vehicular Technology Conference. His research 
interests include radio resource management in modern radio communication systems, scheduling techniques and subcarriers allocation, interference management and cancellation in OFDMA and CDMA systems, physical implementation of OFDMA-based radio communications sys- tems, design of cellular networks, MIMO techniques as well as signal processing, security in wireless systems, radio communication systems design for public safety and military applications, wireless monitoring and transport systems telematics. 